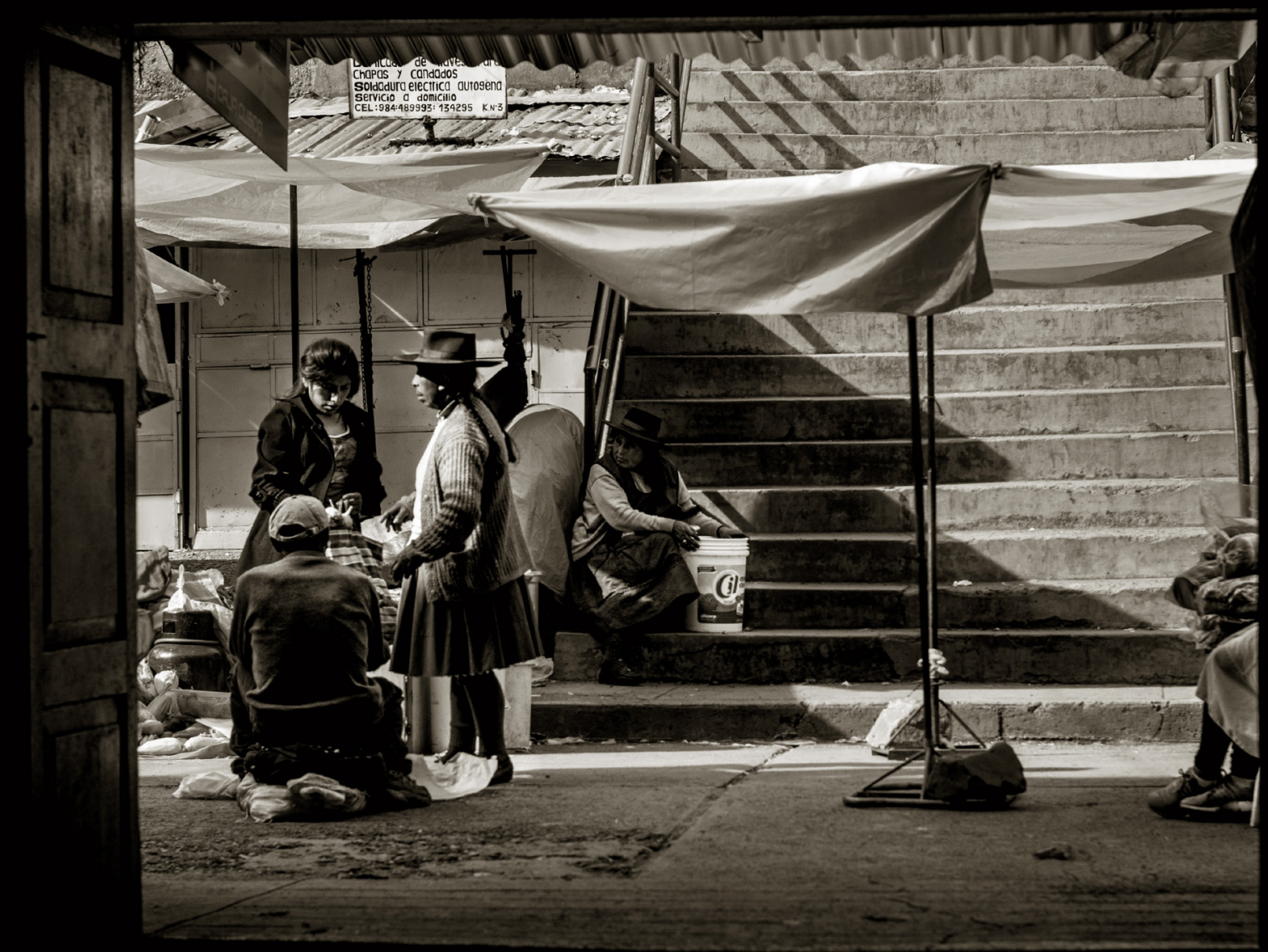

\title{
ROSASPATA
}

\section{UNA MIRADA AL MERCADO \\ CUSQUEÑO A TRAVÉS \\ DE LAFOTOGRAFÍA Y EL \\ PAISAJE SONORO}

Sharon Gonzales Parra Estudiante de la Carrera de Comunicación y Fotografía - UPC

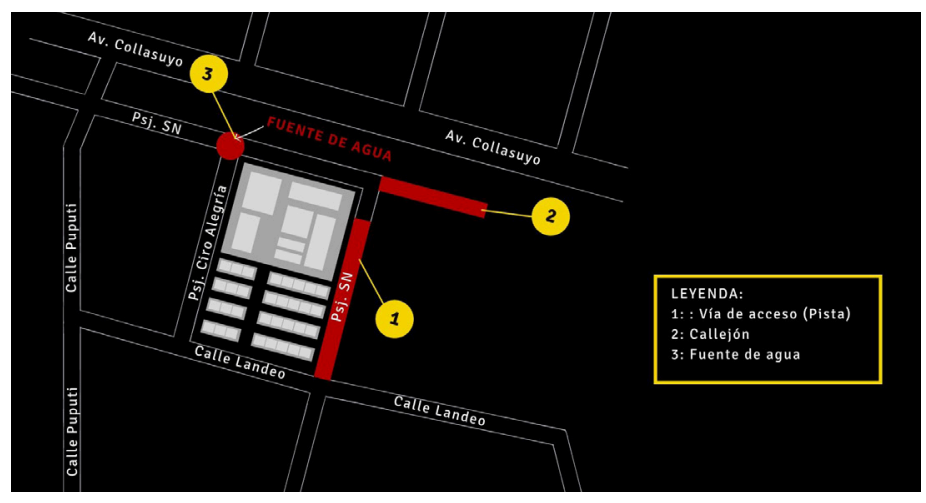

osaspata es un pequeño mercado ubicado muy cerca del Centro Histórico de Cusco y alberga, aproximadamente, a 50 productores y vendedores de la ciudad y de las zonas rurales. Cada sección se encuentra zonificada, por lo que sus 'habitantes' -la mayoría mujeres con hijos pequeños- construyen y afianzan sus lazos amicales a partir de la pertenencia a una determinada sección.

Esta exploración se ubica dentro del campo de la antropología visual y busca encontrar cómo los actores sociales de Rosaspata habitan los espacios heterotópicos. Para Foucault, la heterotopía tiene como regla yuxtaponer en un lugar real varios espacios que normalmente serían, o d o deberían, ser incompatibles. A través de la observación participante busqué descubrir cómo se constituyen dichos subespacios, los mismos que generan diversos usos e interacciones superpuestas.

Al usar de la fotografía, el registro sonoro y la técnica de la foto-elicitación se aborda el espacio con una metodología multisensorial que permite entender sus usos sociales vinculados al sentido de comunidad a partir de tres zonas específicas del mercado.
ESCANEA EL CÓDIGO

QR PARA ACCEDER AL

PAISAJE SONORO. 


\section{VÍA DE ACCESO}

El primer espacio analizado fue la vía de acceso hacia Rosaspata. Al inicio puede observar que dicho espacio fue apropiado por un grupo de mujeres que practicaban una danza para una celebración específica. En una segunda visita,encontré a un pequeño niño, durmiendo sobre algunos sacos de verduras. $Y$ en una tercera visita, el mismo espacio había sido apromeres de una zona rural, que vendían las flores que habían sembrado. Se evidencia entonces una multifuncionalidad espacial.

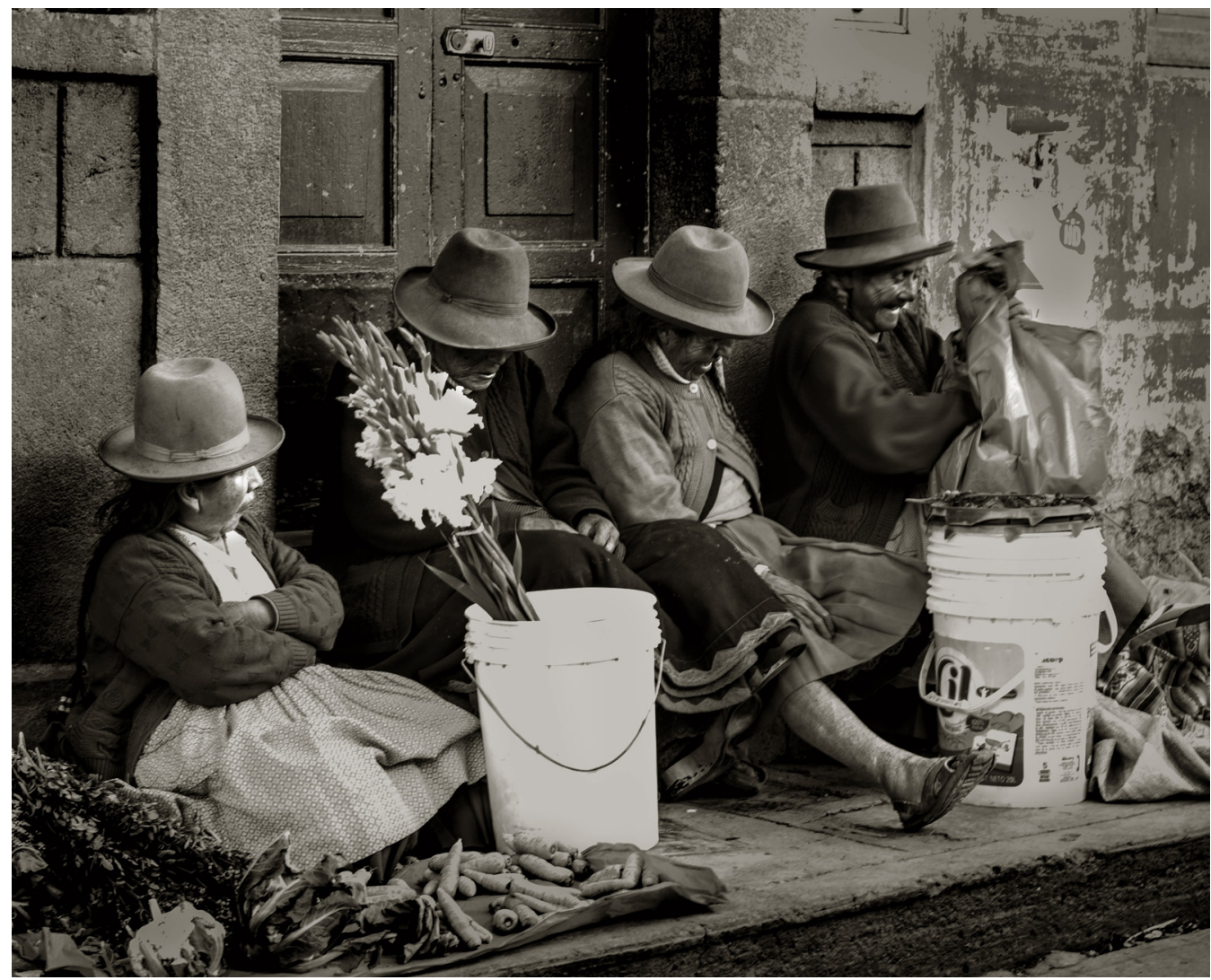

CALLEJÓN

El segundo espacio representa una yuxtaosición incompatible. El callejón, que se ubica en la parte superior derecha del mercado, presenta características inhóspitas y poco hapresentes. Sin embargo, es el lugar de reunión elegido por los niños, hijos de las vendedoras, quienes, a pesar de que su tectónica -que en teoría no permite realizar ninguna actividadhan convertido aquel no lugar (Augé, 2000) en el lugarcotidiano de juegos y fantasías.

En ese sentido, la sonoridad del espacio es rica y compleja. Por lo que decidí realizar un paisaje sonoro. "Los paisajes no solo son vistas hermosas, sino que comprometen toda una experiencia sensorial íntima" (Bender, 2001). Fue gracias a la exploración sonora que pude conocer a los nin ron abiertos para explicarme, con sus palabras, texplicarme, con sus palabras, cómo se constituía ese sentido de comunidad y pertenencia a partir de experimentar el espacio en camaraderí A la vez cada uno de ellos concebía de manera muy distinta el espacio y diferían de la significaciones otorgadas por las personas mayores. Fue justamente esta suerte de apropiación espacial dada por I un hecho que decidí evidenciar a través de la fotografía.

En este punto realicé retratos a todo los iguiente, los llevé impresos para entregárselos uno a uno, junto con sus padres. Más allá de la emotividad y alegría que les proporcionó a los niños salir en una fotografía, puede observar cómo ellos se miraban a través del espacio. Reconocían el lugar de juegos y los diversos objetos del callejón. Este punto fue crucial para darme cuenta de su relación, no solo con dicho espacio, sino también con todo el mercado.
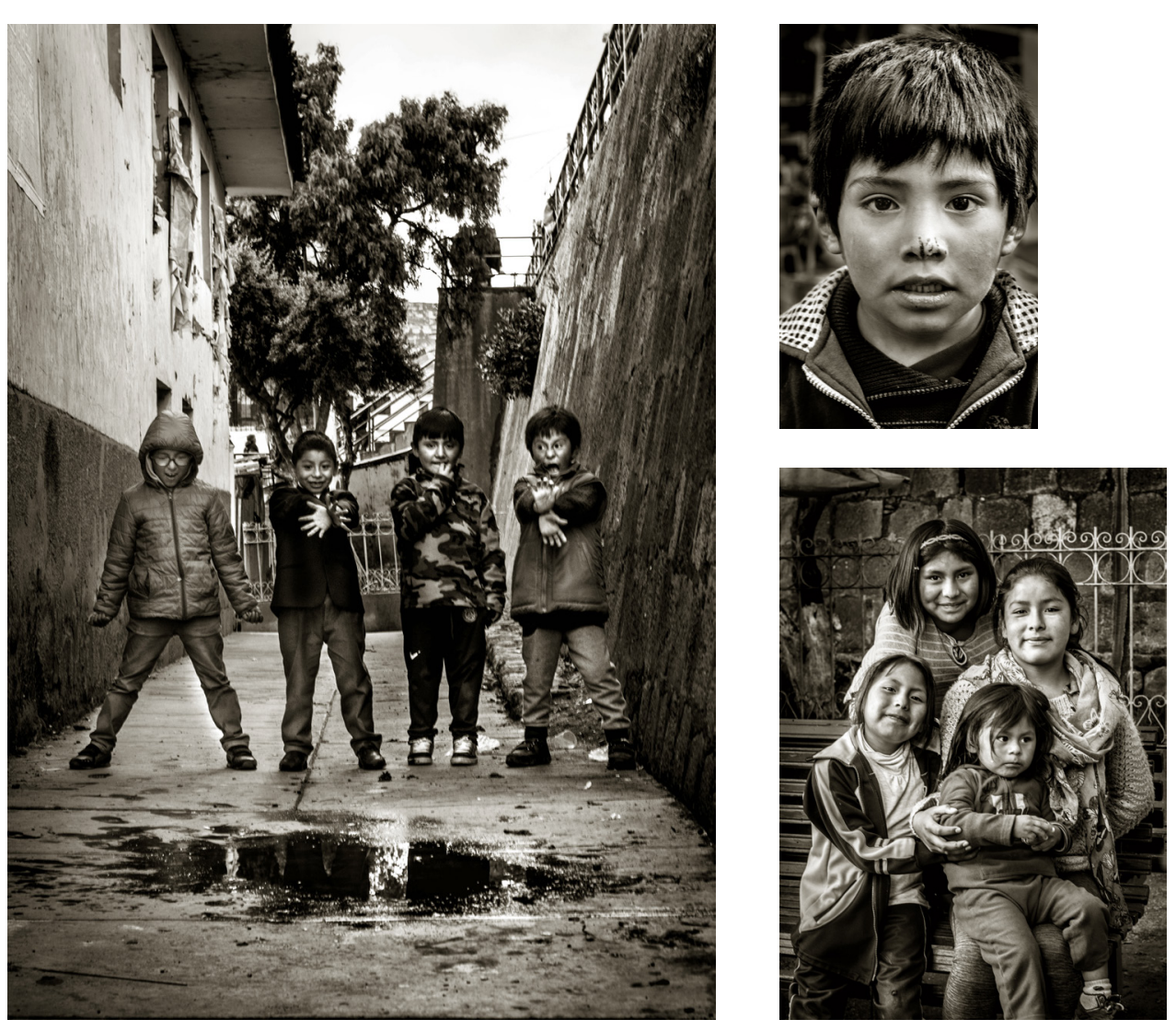
Finalmente, el tercer espacio analizado fue la fuente de agua. Ubicada en la parte superior izquierda del mercado, es el espacio de mayor afluencia de todo el complejo, ya que las vendedoras acuden a esta fuente para hacer el aseo de sus productos y utensilios. El agua de la fuente proviene de un manantial y fluye todos los días del año sin parar.

Este espacio está muy ligado a mi memoria, ya que hace muchos años, cuando yo vivía en la ciudad del Cusco la empresa que brindaba el servicio de agua potable comunicó a toda la ciudad que no habría servicio durante varios días. La desesperación por encontrar agua fue extrema y recuerdo que mi madre me llevó a la fuente del mercado con baldes para cargar agua a casa.

Preguntar en mis entrevistas por el sentido de la fuente también representó un disparador de memoria para las personas que confluyen en el mercado. Muchas de ellas incluso guardan ecto. Una de las vendedoras mencionó que, en el tiempo en que era ambulante, y los fiscalizadores municipales estaban por decomisar su mercadería, ella la escondió al lado de la fuente para que así no se la llevasen, ya que, para ella, representa un espacio seguro. Para un simbolismo oculto en sus aguas. Una mujer comentó que el agua que sale de la fuente es pura porque "estamos en gracia de Dios, y que si nosotros no somos agradecidos las siete plagas del apocalipsis saldrán emanando sangre de aquella fuente."

Resulta interesante contrastar el punto en el que un objeto público puede tomar diferentes significados para las personas, pero que más allá de la subjetividad de nuestros recuerdos; este objeto funciona como un espacio de comunión y encuentro, donde las mujeres se juntan a lavar y crean así también nuevos vínculos que luego se convertirán en memorias. Así asevero finalmente lo que ya mencionaba Heidegger: "Nos encontramos en un universo objetual. Universo que contiene una alta carga de emotividad, experiencia y memoria" (Heidegger,
A través de la mirada hacia la comunidad me pude dar cuenta que las maneras de habitar el espacio son incontables. La pregunta planteada originalmente ¿cómo los habitantes de Rosaspata habitan los espacios heterotópicos del mercado? me permitió abordar de manera en comunidad que se desarroll de manera cotidiana.

La experiencia etnográfica multisensoria me posibilitó mirar -y escuchar- esta comuninalidad del espacio, el cual cobra significancia utilidad y memoria para los diversos habitantes de Rosaspata.

Los entramados sociales no existirían si no fuese por las relaciones que se desprenden de la utilización del espacio y el sentido que los habitantes le dan, albergando una comunidad entrópica con espacios propios a su misma naturaleza.

\section{REFERENCIAS BIBLIOGRÁFICAS}

Augé, M. (2000). Los no lugares. Espacios del anonimato. Gedisa: España.

Bender, B. and Winer, M. (eds) 2001. Contested Landscapes: Movement, Exile and Place. Oxford: Berg.

Foucault, M. (1984). Topologías (Dos conferencias radiofónicas). Obtenido de https://www.mxfractal.org/articulos/ RevistaFractal48MichelFoucault.php

Heidegger, M. (1994). La cosa. Obtenido de http://www.bolivare.unam. mx/cursos/ TextosCurso10-1/HEIDEGGER-\%20LA\%20 cosA.pdf

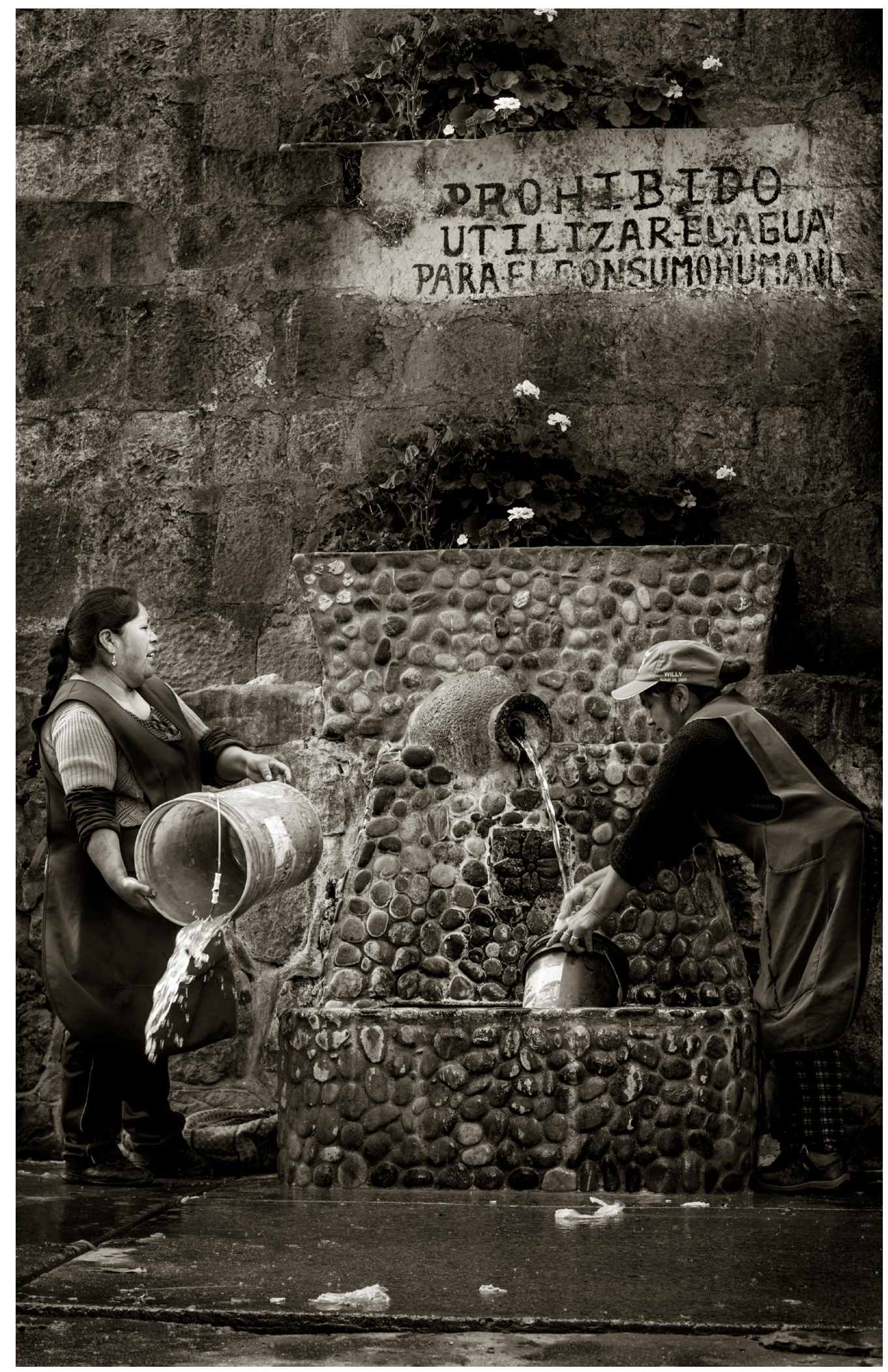

